\title{
Análisis de información y factores de desempeño ambiental y de economia circular en empresas peruanas
}

\section{Analysis of information and factors of environmental performance and circular economy in Peruvian companies}

Julio Hernández Pajares ${ }^{a}$

0000-0002-7481-2912

Valeria Yagui Nishii ${ }^{\mathbf{b}}$
$0000-0002-7901-6261$

Universidad de Piura, Campus Lima, Perú.

a julio.hernandez@udep.edu.pe

bvaleria.yagui.n@gmail.com

\section{Resumen}

Las empresas peruanas con importante impacto de sostenibilidad presentan un significativo desarrollo en su información ambiental. En función de esto, esta investigación tiene como objetivo analizar el nivel de divulgación de información de desempeño ambiental de acuerdo con la Global Reporting Initiative (GRI) y las prácticas de economía circular (EC). De igual forma, determinar su relación con el tamaño y sector empresarial para aquellas empresas que publicaron su reporte de sostenibilidad, y analizar los resultados en el contexto de las teorías de legitimidad e institucional. Se realizó una investigación cuantitativa mediante un análisis de contenido de los reportes de sostenibilidad para determinar los índices del nivel de divulgación de dicha información, descripción y relación de las variables señaladas. Este nivel de divulgación de información ambiental no se cumple en todos los aspectos de la GRI. De hecho, los resultados señalaron un mayor cumplimiento de divulgación de indicadores de gestión ambiental de residuos, agua, transporte y cumplimiento regulatorio, se observó una influencia institucional por tipo de actividad empresarial. Por otro lado, las prácticas de EC tampoco presentaron un alto grado de divulgación, mientras que las prácticas de reducción de consumo de insumos y gestión de residuos mostraron mayor revelación. El sector empresarial presentó relación con el nivel de información ambiental, sin embargo, ni el tamaño ni el sector de las empresas se relacionaron con la información de EC.

Palabras clave: Economía circular; Global Reporting Initiative; información ambiental; reporte de sostenibilidad.
Recibido: 09/01/2021

Aceptado: 20/02/2021

Pub1icado: 17/03/2021

\begin{abstract}
Peruvian companies with significant sustainability impact have a significant development in their environmental information. Based on this, this research aims to analyze the level of disclosure of environmental performance information according to the Global Reporting Initiative (GRI) and circular economy (CE) practices. Similarly, determine its relationship with the size and business sector for those companies that published their sustainability report, and analyze the results within the framework of legitimacy and institutional theories. A quantitative investigation was carried out by means of a content analysis of the sustainability reports to determine the indices of the level of disclosure of said information, description and relationship of the variables indicated. This level of environmental disclosure is not met in all aspects of GRI. In fact, the results indicated greater compliance in the dissemination of environmental waste management, water, transport and regulatory compliance indicators, an institutional influence by type of business activity was observed. On the other hand, CE practices also did not show a high degree of disclosure, while input consumption reduction and waste management practices showed greater disclosure. The business sector presented a relationship with the level of environmental information, however, neither the size nor the sector of the companies were related to the $\mathrm{CE}$ information.
\end{abstract}

Keywords: Circular economy, Global Reporting Initiative, environmental report, sustainability report. 


\section{Introducción}

En la actualidad los reportes de sostenibilidad son un elemento importante para las organizaciones; ya que les permiten divulgar a sus grupos de interés su desempeño ambiental y social como parte de sus objetivos estratégicos. Asimismo, pueden obtener una ventaja competitiva aumentando la eficiencia de los procesos y productos en su impacto ambiental, y mejorando la confianza con las partes interesadas. De esta manera, logran la lealtad de clientes, proveedores, colaboradores y mejorando el acceso a mercados para financiamiento (Ernst \& Young, 2016; Landrum y Ohsowski, 2018; Moneva et al., 2019).

La práctica más difundida de reportes de sostenibilidad ha sido el modelo de la Global Reporting Initiative (GRI). En efecto, diversas investigaciones a nivel mundial encuentran una mayor difusión de estos reportes bajo este estándar en empresas cuyas actividades presentan impacto de sostenibilidad más significativo y con marcada presencia en mercados de valores (AlonsoAlmeida et al., 2015; Landrum y Ohsowski, 2018; Marimon et al., 2012).

Aparte de ello, algunos estudios de organizaciones de economías emergentes, señalan que la influencia institucional en el reporte de sostenibilidad ha sido relevante por parte de organizaciones no gubernamentales internacionales como las Naciones Unidas o Global Reporting Initiative, en mayor medida que los entes reguladores gubernamentales. No obstante, este reporte voluntario, en algunos casos, no ha resultado ser una herramienta efectiva para conocer todas las necesidades de las empresas y las de sus grupos de interés en sus desempeños de sostenibilidad (Ali y Frynas, 2018; Alonso-Almeida et al., 2015; Moneva et al., 2019; Orazalin y Mahmood, 2019).

En Latinoamérica y Perú, las indagaciones evidencian que las empresas con mayor impacto ambiental y social en sectores como el minero, energía, industrial y agroindustria con una importante influencia institucional de organizaciones locales y transnacionales han desarrollado una importante información de sostenibilidad, pero que no llega a los niveles de los estándares internacionales (Alonso-Almeida et al., 2015; Calixto, 2013; Del Barco et al., 2018; Hernández-Pajares, 2018; Moneva et al, 2019; Rabasedas, 2018).
Por otro lado, las estrategias y prácticas de economía circular (EC) son un tema relevante para las organizaciones y gobiernos por representar una alternativa viable al modelo lineal de producción. Esto les permite lograr un compromiso entre productividad y utilización de recursos de forma circular a través de múltiples fases (Bocken et al., 2016; Moneva et al., 2018; Stewart y Niero, 2018; Yuan et al., 2006). Aunque a nivel mundial esta práctica se encuentra muy difundida e investigada, en el Perú, aún está en vías de desarrollo por las empresas y requiere de incentivo gubernamental (Geng et al., 2012; Ghisellini et al., 2016). En ese sentido, a través del Decreto Supremo No 003-2020 del gobierno peruano existe la "Hoja de ruta hacia una Economía Circular en el sector industria" para incentivar la sostenibilidad ambiental en las actividades económicas, promover la EC en los mercados y la generación de soluciones sostenibles para el desarrollo productivo empresarial. Por ello, surgen estudios en el Perú que proponen a la EC como una alternativa de contribución al desarrollo sostenible mediante una mejora en la gestión de la calidad y ecoeficiencia en la industria (AlvarezRisco et al., 2020; Laso et al., 2018).

A partir de los señalado y, con el fin de aportar a la literatura académica en esta área de investigación en Latinoamérica, el presente estudio tiene como objetivo analizar el nivel de divulgación de información de desempeño ambiental de acuerdo con la Global Reporting Initiative (GRI) y las prácticas de economía circular (EC) y su relación con el tamaño y sector empresarial para empresas peruanas que han publicado su reporte de sostenibilidad en el año 2017 en el contexto de las teorías de legitimidad e institucional de reporte ambiental.

La estructura del presente trabajo comprende además de esta introducción, el marco teórico sobre desempeño y reporte ambiental, así como las prácticas de EC. Posteriormente, se propone el diseño metodológico y, finalmente, se expone el análisis de resultados, discusiones y conclusiones.

\section{Marco teórico}

\section{Teorías sobre reporte medioambiental}

Las teorías de legitimidad e institucional, son las más usadas para explicar las motivaciones de las organizaciones para el reporte ambiental y de sostenibilidad, (Cormier et al., 2005; Orazalin 
y Mahmood, 2019). Esta preocupación de las empresas por comunicarse de manera efectiva con las partes interesadas, mejorar la reputación corporativa y justificar su legitimidad en la sociedad sobre su desempeño de sostenibilidad de forma más amplia es una de las causas de la vasta difusión de los reportes de sostenibilidad en el mundo (Alonso-Almeida et al., 2015; Landrum y Ohsowski, 2018; Marimon et al., 2012; Orazalin y Mahmood, 2019).

Teniendo en cuenta la primera de estas teorías, la divulgación ambiental voluntaria es una forma de legitimar la existencia continua y operaciones de las empresas con sus diversas partes interesadas, respondiendo a sus obligaciones con la sociedad, mediante una imagen positiva de desempeño ambiental. Esta tendencia se presenta con mayor incidencia en empresas con impacto en el medio ambiente (Cormier et al., 2005; Deegan, 2002; Husillos, 2007; Simoni et al., 2020). En ese sentido, esta búsqueda de legitimidad tiene como objetivo incidir en las percepciones y expectativas sociales, respaldar la reputación ambiental o desviar la atención de impactos ambientales negativos con los grupos de interés (Deegan, 2002; Llena et al., 2007). A propósito de este objetivo, estudios recientes ponen de manifiesto que las empresas responden con ello ante la presión e influencia de los grupos de interés, y buscan cambiar sus expectativas con una información positiva (Mio et al., 2020; Simoni et al., 2020).

Sobre la base de la teoría institucional, se comprende que las empresas desarrollen un comportamiento informativo similar a otras empresas (isomorfismo) o que se desempeñen de acuerdo con lo que la normativa exige por influencia de asociaciones empresariales y profesionales, entes reguladores locales $\mathrm{u}$ organizaciones no gubernamentales (Naciones Unidas, GRI, International Organization for Standardization). Con la influencia de sus estándares (Pacto Mundial, GRI Standards, ISO 26000) promueven una divulgación de información armonizada similar a las empresas de sus sectores empresariales y, responden a la presión de grupos de interés (Aerts et al., 2006; De Villiers y Alexander, 2014; Martínez-Ferrero y García-Sánchez, 2017; Neu et al., 1998). Sin embargo, esta influencia no ha logrado que estos reportes constituyan una rendición de cuentas $y$, gestión y comunicación más efectiva (Bebbington et al., 2012; Larrinaga et al., 2002).
Las investigaciones sobre influencia institucional siguen vigentes y las más recientes, señalan una influencia de los modelos de estándares internacionales, regulaciones para determinadas industrias, presión de sectores empresariales en el reporte ambiental y búsqueda de mejorar la imagen pública, por lo que también resulta manifiesta la teoría de legitimidad (Khan et al., 2020; Madein, 2020).

\section{Factores de reporte ambiental}

Además de las teorías revisadas que explican el reporte ambiental, los estudios señalan factores corporativos determinantes en la divulgación en el marco de estas teorías. De esta manera, las empresas de mayor tamaño y desempeño están incentivadas a revelar una mayor información de sostenibilidad. Esto se debe a la significativa influencia institucional y búsqueda constante de legitimidad, pues es innegable que existe no solo una presión por parte de la sociedad y de los grupos de interés de rendir cuenta sobre este tipo de desempeño, sino también una mayor disposición de recursos y menores costos para elaborar dicha información (Da Silva y Aibar, 2011; D'Amico et al., 2016; Orazalin y Mahmood, 2019; Wirth et al., 2016).

Respecto al sector, la naturaleza de las actividades de las empresas resulta ser determinante en el tipo y nivel de información ambiental, debido al nivel de impacto en el medio ambiente. Esto es válido, sobre todo, para las del rubro de energía, minería e industria, que tienen más que justificada la necesidad de legitimar sus actividades frente a la sociedad (Baalouch et al., 2019; Bednárová et al., 2019; Cormier y Magnan, 2003; Gallego-Álvarez et al., 2018; Orazalin y Mahmood, 2019).

De acuerdo con lo señalado anteriormente proponemos las siguientes hipótesis:

H1: El sector empresarial incide en el nivel de cumplimiento de información ambiental.

H2: El sector empresarial y tamaño se correlacionan positivamente con el nivel de información ambiental.

\section{Prácticas de economía circular}

A pesar de que los estudios sobre EC se han centrado en la proposición de modelos de negocio 
con esta propuesta y en las prácticas respectivas en las organizaciones, la investigación de la EC como estrategia empresarial aún se encuentra en desarrollo (Aranda-Usón et al., 2020). Sus objetivos son la reducción del flujo de materiales, la eficiencia energética, la renovación constante del capital natural y social en múltiples fases y gestionar ciclos de materiales de alto valor en lugar del reciclaje tradicional (Ghisellini et al., 2016; Yuan et al., 2006). La EC se representa, generalmente, en la literatura como una combinación de actividades con distintitos enfoques y no como uno sistémico. Esto significa que no todas las definiciones muestran vínculos explícitos con la de sostenibilidad, aunque la EC se relaciona con el impacto ambiental, no se encuentra una correspondencia de su concepto con el de desempeño social y desarrollo sostenible o producción eco-innovadora sostenible (Kirchherr et al., 2017; Prieto-Sandoval et al., 2018).

Por otro lado, el modelo de indicadores de la GRI en temas ambientales ha servido de base para la medición de aspectos de desarrollo de EC como los estudios de Li et al. (2010) y Veleva et al. (2017). De acuerdo con la literatura, la Tabla 1 muestra distintos aspectos de desempeño de EC que se consideran en esta investigación.

Tabla 1.

Aspectos de EC de acuerdo a la literatura.

\begin{tabular}{|c|c|}
\hline Aspectos de EC & Autores \\
\hline $\begin{array}{l}\text { Reducción de insumos/recursos y } \\
\text { menor utilización de recursos } \\
\text { naturales }\end{array}$ & $\begin{array}{l}\text { Cerdá y Khalilova (2016); Elia et al. (2017); Geng et al. (2012); } \\
\text { Kirchherr et al. (2017); Potting et al. (2017); Bocken et al. } \\
\text { (2016); Ying y Li-jun (2012) }\end{array}$ \\
\hline Reducción de emisiones & $\begin{array}{l}\text { Cerdá y Khalilova (2016); Elia et al. (2017); Geng et al. (2012); } \\
\text { Stewart y Niero (2018). }\end{array}$ \\
\hline $\begin{array}{l}\text { Disminuir la generación de materiales } \\
\text { y de residuos }\end{array}$ & $\begin{array}{l}\text { Cerdá y Khalilova (2016); Geng et al. (2012); Kirchherr et al. } \\
\text { (2017); Ying y Li-jun (2012) }\end{array}$ \\
\hline $\begin{array}{l}\text { Reemplazar los recursos no } \\
\text { renovables por renovables }\end{array}$ & $\begin{array}{l}\text { Cerdá y Khalilova (2016); Moneva et al. (2018); Ghisellini et al. } \\
\text { (2016); Bocken et al. (2016); Ying y Li-jun (2012); Elia et al. } \\
(2017)\end{array}$ \\
\hline $\begin{array}{l}\text { Mayor proporción de materiales } \\
\text { reciclados que reemplacen a } \\
\text { materiales vírgenes }\end{array}$ & $\begin{array}{l}\text { Cerdá y Khalilova (2016); Elia et al. (2017); Ying y Li-jun } \\
\text { (2012); Stewart y Niero (2018). }\end{array}$ \\
\hline $\begin{array}{l}\text { Reutilización/reciclaje de los residuos } \\
\text { generados }\end{array}$ & $\begin{array}{l}\text { Cerdá y Khalilova (2016); Moneva et al. (2018); Elia et al. } \\
\text { (2017); Geng et al. (2012); Ghisellini et al. (2016); Kirchherr et } \\
\text { al. (2017); Potting et al. (2017); Bocken et al. (2016); Aranda- } \\
\text { Usón et al. (2020); Stewart y Niero (2018); Singhal et al. } \\
\text { (2020). }\end{array}$ \\
\hline Eco Diseño de Procesos & $\begin{array}{l}\text { Cerdá y Khalilova (2016); Moneva et al. (2018), Elia et al. } \\
\text { (2017); Ghisellini et al. (2016); Kirchherr et al. (2017); Potting } \\
\text { et al. (2017); Bocken et al. (2016); Ying y Li-jun (2012); } \\
\text { Aranda-Usón et al. (2020); Scarpellini et al. (2020b). }\end{array}$ \\
\hline
\end{tabular}

Estudios más recientes señalan distintos factores en los sistemas y prácticas de EC, capacidades como las prácticas de desempeño ambiental, responsabilidad social empresarial, políticas de contabilidad ambiental y demandas de los grupos de interés se relacionan con el alcance de EC de las empresas y la eco innovación (Scarpellini et al., 2020a, 2020b). En efecto, las motivaciones, el liderazgo y compromiso de los directivos, propietarios y accionistas de las empresas y, la influencia de los grupos de interés son los factores que inciden en la implementación de sistemas de EC (Chiappetta, et al., 2020; Moktadir et al., 2020; Singhal, 2020).
Diversas investigaciones buscan proporcionar modelos de gestión y control a las empresas y marcos de políticas públicas para los gobiernos para incentivar y regular los sistemas de EC (Scarpellini et al., 2020b; Singhal, 2020). Así mismo, señalan la necesidad de una capacitación en las empresas, de legislaciones claras y apoyos económicos públicos para la implementación de estos sistemas de EC (Chiappetta et al., 2020; Moktadir et al., 2020).

De acuerdo con los antecedentes revisados anteriormente, proponemos las siguientes hipótesis: 
H3: El sector empresarial incide en el nivel de información de aspectos de EC.

H4: El sector empresarial y tamaño se correlacionan positivamente con el nivel de información de aspectos de EC.

\section{Metodología}

\section{Diseño y técnicas}

Este estudio cuantitativo de naturaleza exploratoria tuvo un alcance descriptivo del nivel de información de los aspectos de desempeño ambiental y de prácticas de EC. Asimismo, analizó la correlación e incidencia entre las variables independientes de tamaño y sector empresarial con las variables dependientes de información de desempeño ambiental y de EC. Además del análisis descriptivo, se realizó un análisis multivariante de correlaciones de Spearman y de incidencia de Kruskal-Wallis para probar las hipótesis de relación entre las variables que no presentan una distribución normal.

\section{Muestra}

El estudio se realizó con una muestra de la población de empresas peruanas, y se seleccionó solo a las que publicaron sus memorias de sostenibilidad en la base de datos de la GRI (Sustainability Disclosure Database) para el periodo 2017, último periodo disponible a la fecha de la investigación. La descripción de las empresas por su sector se muestra en la Tabla 2, en los cuales los sectores de industria, energía y minería resultaron los más significativos y con importante impacto ambiental. De las empresas, se revisaron para 58 sus reportes bajo la Guía G4 de la GRI y para 9 sus reportes de sostenibilidad presentadas bajo otro modelo no GRI, obtenido de la base de datos o de la página web corporativa.

Tabla 2.

Muestra de empresas por sector.

\begin{tabular}{lll}
\hline & Frecuencia & Porcentaje \\
\hline Energía & 12 & $18 \%$ \\
Minería & 11 & $16 \%$ \\
Industria & 10 & $15 \%$ \\
Financiero & 10 & $15 \%$ \\
Servicios & 10 & $15 \%$ \\
Comercial & 6 & $9 \%$ \\
Inmobiliario/Construcción & 5 & $8 \%$ \\
Agroindustria & 3 & $4 \%$ \\
\hline Total & $\mathbf{6 7}$ & $\mathbf{1 0 0} \%$ \\
\hline
\end{tabular}

\section{Variables}

La primera variable dependiente comprende el nivel de cumplimiento de divulgación ambiental. Para ello, se verificó el cumplimiento de información de los 34 indicadores de la Guía G4 de la GRI, clasificados en las categorías de gestión de materiales, agua, biodiversidad, emisiones, residuos, productos-servicios, cumplimiento regulatorio, transporte, evaluación ambiental de proveedores y mecanismos de reclamación. Para el análisis descriptivo, cada indicador se midió con una variable categórica con valor 0 si no revela información y con valor 1 si revela.

Se obtuvieron promedios de incidencia para determinar los índices de cumplimiento de las categorías de desempeño ambiental para el análisis multivariante (Rabasedas, 2018). En cuanto a la segunda variable dependiente que correspondía a los aspectos de prácticas de EC revelados por las empresas en sus memorias o reportes de sostenibilidad (Stewart y Niero, 2018), se revisó su cumplimiento mediante un análisis de contenido de los aspectos determinados de acuerdo con la literatura revisada (Ver Tabla 1). Para evaluar el nivel de revelación de aspectos de EC se determinó una variable categórica de valor 0 sino no revela sobre el aspecto, 1 si revela.

Respecto a las variables independientes, el tamaño de la empresa se midió mediante el número de trabajadores revelados en el reporte (Alonso-Almeida et al., 2015) y, para el análisis multivariante, se consideró el logaritmo natural del número de trabajadores con el fin de disminuir su dispersión. En cuanto a la clasificación por industria, se consideraron los de la Bolsa de Valores de Lima: agroindustria, comercial, industrial, inmobiliario/construcción, energía, financiero, minería y servicios para el análisis descriptivo, y una variable categórica de clasificación en dos grupos, uno de valor 1 para empresas con impacto de sostenibilidad importante (agroindustria, industrial, inmobiliario/construcción, energía; minería) y con valor 0 para las del grupo que no tenían dicho impacto (comercial, financiero y servicios) para el estudio de correlación (Jackson et al., 2019).

\section{Resultados y discusión}

\section{Análisis descriptivo}


En la Tabla 3, se muestran las empresas estudiadas de la base de datos del GRI con su promedio de porcentaje de incidencia de los indicadores ambientales de la Guía del GRI y de los aspectos de desempeño de EC informados en sus memorias. De acuerdo con los indicadores ambientales, las empresas del sector minero, energía, industria y construcción presentaron un porcentaje mayor al $50 \%$ de cumplimiento. Al respecto, cabe resaltar que para algunas empresas no aplican todos los desempeños ambientales de la guía del GRI por la naturaleza de sus operaciones, y estos son mayores para las empresas con un marcado impacto ambiental que para las de servicios o financieras.
Respecto al cumplimiento de indicadores de EC, no destaca algún sector específico con mayor índice y no se encuentra correlación entre ambos niveles de cumplimiento de información. De hecho, se hallaron empresas con un alto nivel de cumplimiento de información sobre prácticas de EC, pero no necesariamente verifican de igual forma los indicadores ambientales. Esto puede explicarse señalando que las empresas no presentan sistemas de EC como parte de las estrategias y prácticas ambientales y de sostenibilidad (PrietoSandoval et al., 2018; Veleva et al., 2017).

Tabla 3.

Empresas con promedio de cumplimiento de indicadores ambientales y aspectos de EC.

\begin{tabular}{|c|c|c|c|}
\hline Empresa & Sector & $\begin{array}{l}\text { Promedio Cumplimiento } \\
\text { Indicadores Ambientales }\end{array}$ & $\begin{array}{c}\text { Promedio de } \\
\text { Incidencia de Aspectos } \\
\text { de EC } \\
\end{array}$ \\
\hline YANACOCHA & Minería & $94 \%$ & $43 \%$ \\
\hline EDEGEL GENERACIÓN & Energía & $82 \%$ & $71 \%$ \\
\hline EDELNOR DISTRIBUCIÓN & Energía & $79 \%$ & $71 \%$ \\
\hline TASA & Industria & $68 \%$ & $29 \%$ \\
\hline ENEL GENERACION PIURA & Energía & $68 \%$ & $29 \%$ \\
\hline JJC CONTRATISTAS & Inmobiliario/Construcción & $59 \%$ & $57 \%$ \\
\hline MINSUR & Minería & $56 \%$ & $86 \%$ \\
\hline MILPO & Minería & $56 \%$ & $57 \%$ \\
\hline PETRÓLEOS DEL PERÚ & Energía & $53 \%$ & $100 \%$ \\
\hline UNICON & Servicios & $50 \%$ & $86 \%$ \\
\hline UNIÓN ANDINA DE CEMENTOS & Industria & $47 \%$ & $86 \%$ \\
\hline ANTAMINA & Minería & $47 \%$ & $57 \%$ \\
\hline BANCO CONTINENTAL PERU & Financiero & $44 \%$ & $71 \%$ \\
\hline EGASA & Energía & $44 \%$ & $57 \%$ \\
\hline CONTUGAS & Energía & $41 \%$ & $57 \%$ \\
\hline TISUR & Servicios & $38 \%$ & $100 \%$ \\
\hline ACEROS AREQUIPA & Industria & $38 \%$ & $71 \%$ \\
\hline SODIMAC - PERU & Industria & $38 \%$ & $57 \%$ \\
\hline FERREYCORP & Comercial & $35 \%$ & $71 \%$ \\
\hline COMPAÑÍA MINERA PODEROSA & Minería & $35 \%$ & $71 \%$ \\
\hline IIRSA NORTE & Inmobiliario/Construcción & $35 \%$ & $57 \%$ \\
\hline SCOTIABANK PERU & Financiero & $35 \%$ & $57 \%$ \\
\hline GOLD FIELDS EN PERÚ & Minería & $35 \%$ & $43 \%$ \\
\hline ELECTRO SUR ESTE & Energía & $35 \%$ & $29 \%$ \\
\hline PACIFICO & Financiero & $32 \%$ & $71 \%$ \\
\hline ANTAPACCAY & Minería & $32 \%$ & $71 \%$ \\
\hline SEDAPAL & Servicios & $32 \%$ & $57 \%$ \\
\hline DANPER TRUJILLO & Agroindustria & $29 \%$ & $71 \%$ \\
\hline CELEPSA PERÚ & Energía & $29 \%$ & $57 \%$ \\
\hline EL BROCAL & Minería & $29 \%$ & $57 \%$ \\
\hline DIRECTV PERÚ & Servicios & $29 \%$ & $57 \%$ \\
\hline TRANSPORTADORA DE GAS DEL PERÚ & Energía & $29 \%$ & $14 \%$ \\
\hline LIMA AIRPORT PARTNERS & Servicios & $29 \%$ & $14 \%$ \\
\hline CEMENTOS PACASMAYO & Industria & $26 \%$ & $71 \%$ \\
\hline GRUPO TELEFÓNICA EN EL PERÚ & Servicios & $26 \%$ & $71 \%$ \\
\hline PRIMA AFP & Financiero & $26 \%$ & $43 \%$ \\
\hline VOLCAN CIA MINERA & Minería & $26 \%$ & $43 \%$ \\
\hline WONG & Comercial & $24 \%$ & $71 \%$ \\
\hline
\end{tabular}


BACKUS Y JOHNSTON

PWC PERU

RANSA

CÁLIDDA

ELECTROPERU

BANCO DE CRÉDITO DEL PERÚ

UNIVERSIDAD DEL PACÍFICO

BUENAVENTURA

GRUPO GRAÑA Y MONTERO

MACISA

CANDELA

COSAPI

PERUVIAN NATURE

MAKRO

RIMS

AUSTRA

CAMPOSOL

LAS BAMBAS

NESTLE PERÚ

INTERBANK

SAN FERNANDO

APC CORPORACIÓN

Fuente: Elaboración propia en base a los datos del estudio.

BACKUS Y JOHNSTON
PWC PERÚ
REPSOL PERÚ
RANSA
CÁLIDDA
ELECTROPERU
BANCO DE CRÉDITO DEL PERÚ
BANCO DE LA NACIÓN
UNIVERSIDAD DEL PACÍFICO
BUENAVENTURA
GRUPO GRAÑA Y MONTERO
IIRSA SUR CONCESIÓN VIAL
MACISA
CANDELA
COSAPI
PERUVIAN NATURE
MAKRO
RIMAC SEGUROS
CREDISCOTIA
LA VIGA
AUSTRAL
CAMPOSOL
LAS BAMBAS
NÉSTLE PERÚ
INTERBANK
ENTEL PERÚ
SAN FERNANDO
SURA PERÚ
APC CORPORACIÓN
PATE EA

\begin{tabular}{ccc} 
Industria & $24 \%$ & $71 \%$ \\
Servicios & $24 \%$ & $57 \%$ \\
Energía & $21 \%$ & $86 \%$ \\
Comercial & $21 \%$ & $71 \%$ \\
Energía & $21 \%$ & $71 \%$ \\
Energía & $21 \%$ & $57 \%$ \\
Financiero & $18 \%$ & $71 \%$ \\
Financiero & $18 \%$ & $71 \%$ \\
Servicios & $18 \%$ & $71 \%$ \\
Minería & $18 \%$ & $71 \%$ \\
Inmobiliario/Construcción & $18 \%$ & $57 \%$ \\
Inmobiliario/Construcción & $18 \%$ & $57 \%$ \\
Comercial & $18 \%$ & $43 \%$ \\
Industria & $15 \%$ & $86 \%$ \\
Inmobiliario/Construcción & $15 \%$ & $71 \%$ \\
Agroindustria & $15 \%$ & $57 \%$ \\
Comercial & $15 \%$ & $57 \%$ \\
Financiero & $15 \%$ & $57 \%$ \\
Financiero & $15 \%$ & $43 \%$ \\
Comercial & $12 \%$ & $71 \%$ \\
Industria & $12 \%$ & $57 \%$ \\
Agroindustria & $12 \%$ & $29 \%$ \\
Minería & $12 \%$ & $14 \%$ \\
Industria & $9 \%$ & $71 \%$ \\
Financiero & $9 \%$ & $57 \%$ \\
Servicios & $9 \%$ & $57 \%$ \\
Industria & $9 \%$ & $43 \%$ \\
Financiero & $9 \%$ & $43 \%$ \\
Servicios & $9 \%$ & $14 \%$ \\
\hline
\end{tabular}

Cómo observamos en la Figura 1, en cuanto al nivel de cumplimiento de divulgación de indicadores de desempeño ambiental, las empresas estudiadas revelaron de mayor forma aspectos de cumplimientos regulatorios ambientales, gestión de agua, residuos y de transporte. La gestión de emisiones, con la biodiversidad y responsabilidad en producto y servicios ofrecidos fueron los de menor observancia. Adicional a ello, se percibió que no se cumplieron en un alto nivel, los aspectos requeridos por la Guía del GRI. Una vez más fue mayor para aquellas organizaciones con importante desempeño con el medio ambiente (Calixto, 2013; Del Barco et al., 2018; Hernández-Pajares, 2018).

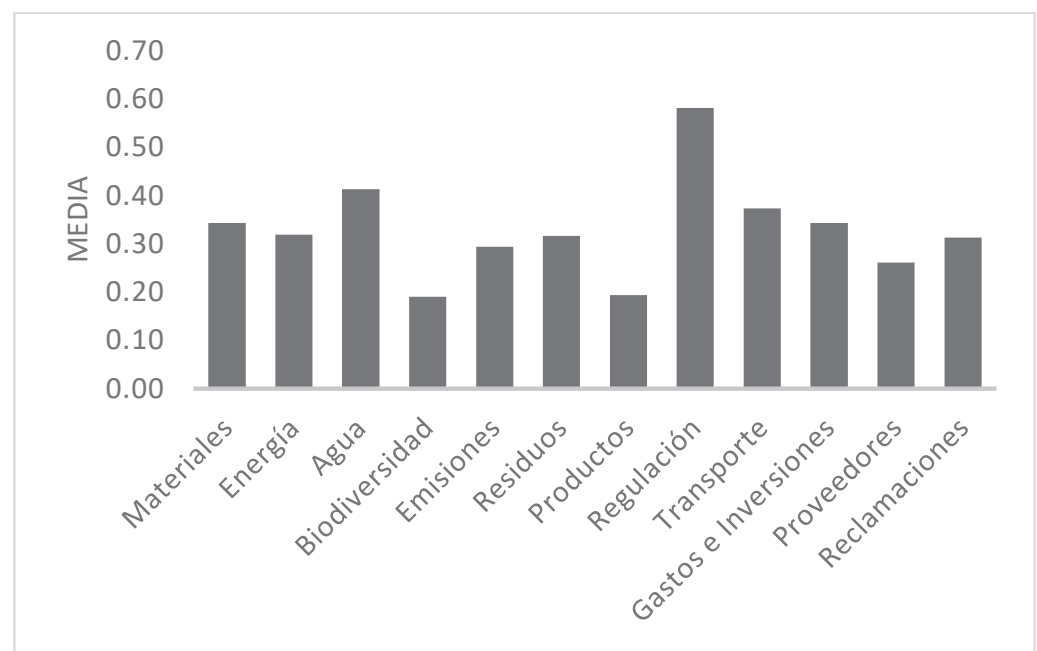

Figura 1. Cumplimiento de categorías ambientales.

Fuente: Elaboración propia. 
Observamos en la Figura 2, que las empresas de construcción, industriales, producción y servicio de energía y minería son las que presentan un mayor índice de cumplimiento promedio de aspectos de desempeño ambiental revelados en sus reportes, debido al impacto en el medio ambiente de sus actividades. Fue claro que el tipo de actividad tuvo una incidencia en la divulgación de información, por una búsqueda de legitimación de sus actividades principalmente para las actividades de energía (generación eléctrica, petróleo y gas) y minería (Bednárová et al., 2019; Cormier y Magnan, 2003; Da Silva y Aibar, 2011; D’Amico et al., 2016; Jenkins y Yakovleva; 2006; Simoni et al., 2020).

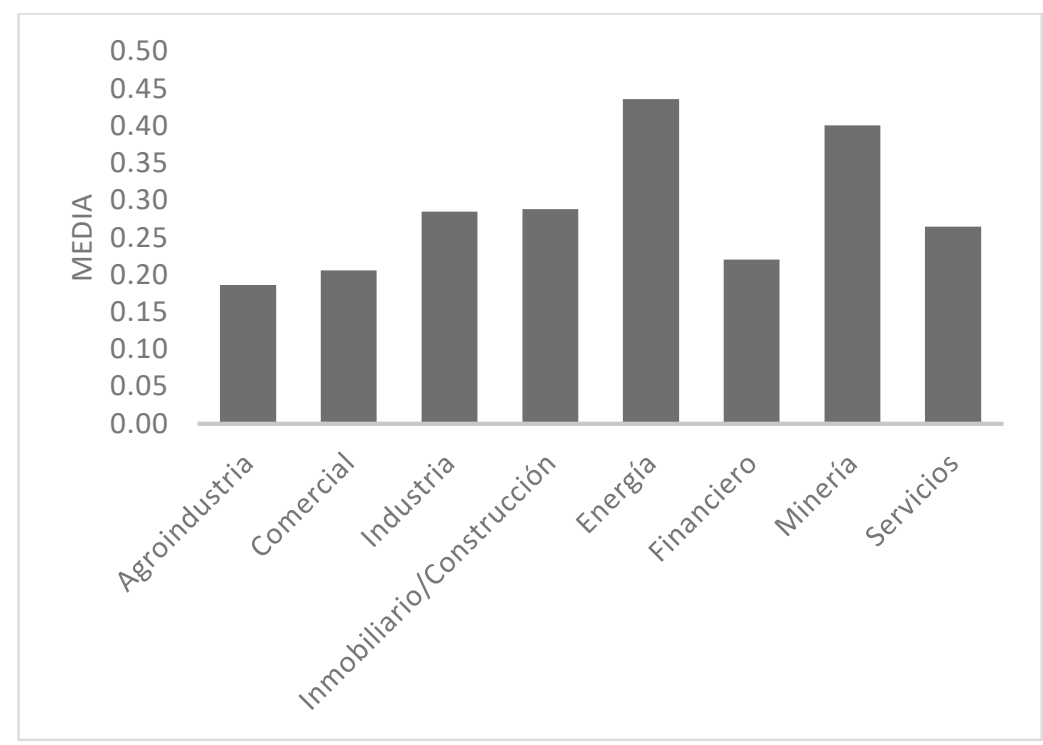

Figura 2. Nivel de información ambiental por Sector.

Fuente: Elaboración propia.

Aunque el nivel de cumplimiento de aspectos de EC no presentó una alta incidencia en todos sus aspectos, como se observa en el Figura 3, se percibió un importante nivel de información para los desempeños relacionados con la reducción de de residuos generados. Estas prácticas son consistentes con los desempeños ambientales de acuerdo con la Guía de la GRI, pero se presentan como informes de impactos o resultados (outputs) a diferencia de los aspectos de la EC que son parte de los procesos como explica Veleva et al. (2017). Cuestiones como cambios a recursos renovables, uso de recurso reciclados y ecodiseño son procesos con menor incidencia y no presentados como un sistema de gestión (Kirchherr et al., 2017).

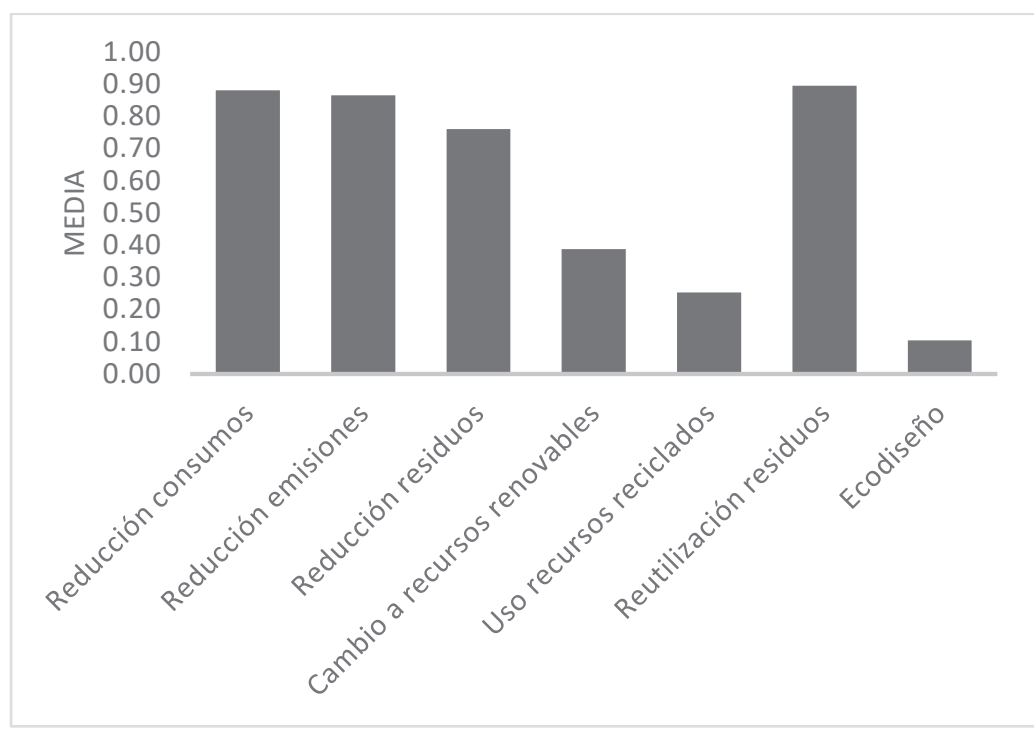

Figura 3. Cumplimiento de Aspectos de EC. 


\section{Análisis multivariante}

En primer lugar, para analizar si el sector empresarial tiene incidencia significativa en los niveles de cumplimiento promedio de desempeño ambiental de acuerdo con los aspectos del GRI y a los aspectos de prácticas de EC aplicamos las pruebas de Kruskal-Wallis.

Los resultados en la Tabla 4 señalan que solamente el sector empresarial incidió significativamente $(p$ $<0,10$ ) en el nivel de cumplimiento de desempeño ambiental de acuerdo con los indicadores de la GRI, por lo que no rechazamos la $\mathrm{H} 1$ para esta variable. Los sectores de actividad industrial, de energía (generación eléctrica, petróleo y gas) y minería presentaron una mayor información de desempeño ambiental. Esto puede explicarse con los estudios que señalan que los sectores con mayor impacto ambiental buscan una legitimación de sus actividades en el ámbito social donde se desenvuelven siendo más evidente en los sectores industriales y minería (Bednárová et al., 2019; Jenkins y Yakovleva; 2006, Mio et al., 2020).

Tabla 4.

Estadísticos de prueba de Kruskal Wallis.

\begin{tabular}{lcc}
\hline & $\begin{array}{c}\text { Índice de Información } \\
\text { Ambiental }\end{array}$ & $\begin{array}{c}\text { Índice de } \\
\text { Información de EC }\end{array}$ \\
\hline $\mathrm{H}$ de Kruskal-Wallis & 13,440 & 2,450 \\
$\mathrm{gl}$ & 7 & 7 \\
$\mathrm{Sig}$. asintótica & $0.062 *$ & 0.931 \\
\hline${ }^{*} \mathrm{p}<0.10, * * \mathrm{p}<0.05$ & & \\
Fuente: elaboración propia & &
\end{tabular}

En el caso del índice de información de aspectos de EC, el nivel de cumplimiento no varió significativamente teniendo en cuenta al sector empresarial, por lo que rechazamos la H3. No se percibió una diferencia significativa entre lo divulgado por cada tipo de actividad ( $p>0,10$; $\mathrm{p}>0,05$ ). Fueron muy pocas las empresas, sin sector específico, las que cumplieron los aspectos de EC. Estos solo estuvieron enfocados en gestión de consumos, residuos y reciclaje, pero no mencionaron específicamente el concepto de EC. Esto se debe a un incipiente desarrollo e implantación de la EC como estrategia empresarial (Moneva et al, 2018).

Finalmente, para evaluar la correlación entre las variables independientes de tamaño medido por número de trabajadores y sector como variable dicotómica (empresas con y sin impacto ambiental significativo) con las variables dependientes de nivel de cumplimiento de aspectos ambientales de la GRI y nivel de información de aspectos de EC, se ha realizado un análisis de correlación de Spearman.

En la Tabla 5, evidenciamos que, para el caso del tamaño de la empresa por el número de trabajadores la relación con algunos aspectos de desempeño ambiental es significativa $(p<0,01$ y $p<0,05)$ pero negativa, por lo que rechazamos la $\mathrm{H} 2$ para esta variable. Estos resultados son similares a algunos autores que encontraron que el tamaño de las empresas no se relaciona positivamente con la información ambiental (Baalouch et al., 2019; Wirth et al. 2016). El número de trabajadores, como variable representativa del tamaño, no se relaciona positivamente con el nivel de información ambiental. Por el contrario, las empresas con menos trabajadores tienen un mayor incentivo de presentar información de gestión de biodiversidad, cumplimiento de regulación, información sobre gastos e inversiones ambientales, y atención de reclamaciones. Incluso se encontraron empresas con menor número de trabajadores con un mayor nivel de información. Este fue el caso de las dedicadas a servicios públicos de energía, gas, telefonía, logística.

Considerando el sector empresarial, la Tabla 5 muestra una relación de forma significativa y positiva $(p<0,01$ y $p<0,05)$ entre el tipo de actividad empresarial con algunos aspectos de desempeño ambiental por lo que no rechazamos la $\mathrm{H} 2$ para esas variables. Es así que de las empresas del grupo de mayor impacto ambiental de la Figura 2, las de agroindustria, industria, energía y minería se relacionaron con un mayor nivel de cumplimiento de información en aspectos de gestión medioambiental (Baalouch et al., 2019; Bednárová et al., 2019; Cormier y Magnan, 2003; Gallego-Álvarez et al., 2018). 


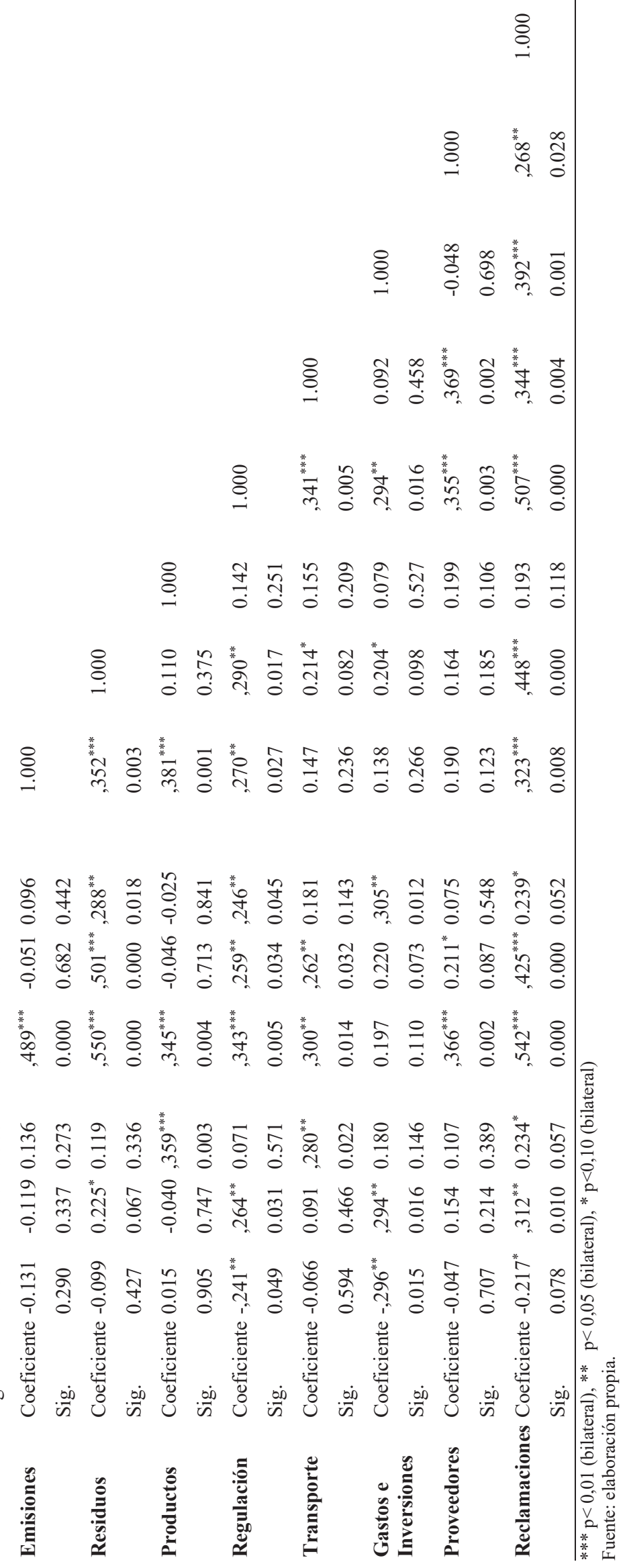

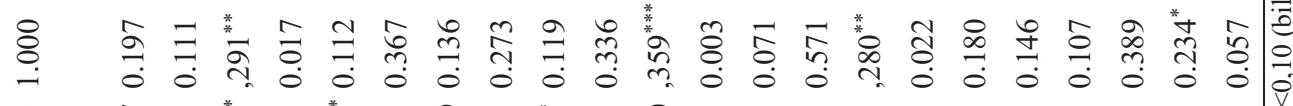

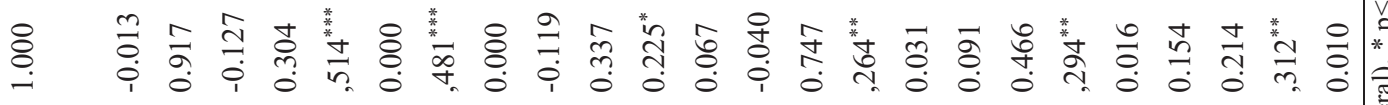

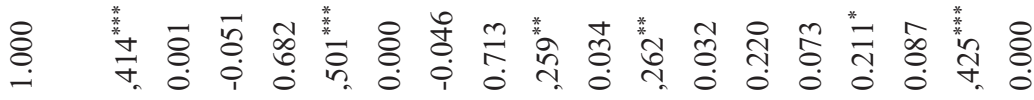

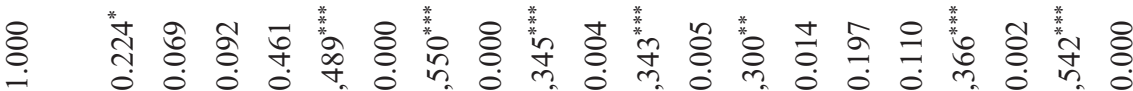

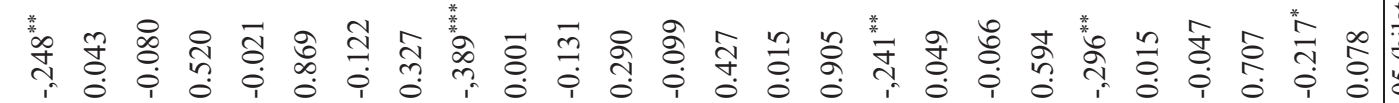

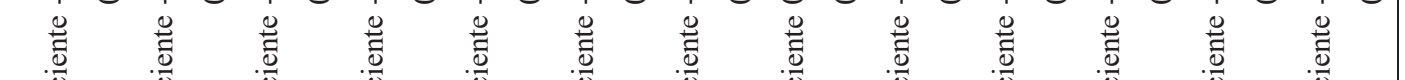
家 
En el estudio se encuentra correlación positiva y significativa entre las empresas de actividad con impacto señalada y la información de gestión de agua, residuos, biodiversidad, cumplimiento regulatorio ambiental y atención de reclamaciones, e información sobre gastos e inversiones ambientales para empresas agroindustriales, de industria, producción de energía y minería. Sectores con influencia significativa de sus actividades en el medioambiente a diferencia de empresas comerciales, financieras y servicios (Alonso-Almeida et al., 2015; Calixto, 2013; Hernández-Pajares, 2018). Una vez más resulta visible el interés de legitimar sus actividades con las comunidades con quienes se relacionan y la búsqueda de mantener relaciones provechosas (Cormier et al., 2005; Deegan, 2002; Husillos, 2007; Mio et al., 2020; Simoni et al., 2020). El seguimiento de los estándares internacionales del GRI, y específicos para determinados sectores empresariales, señalaron una influencia institucional y un isomorfismo de seguir modelos similares de información de acuerdo con la actividad empresarial, como el caso de los sectores mineros, industrial y energía, por ejemplo (Aerts et al., 2006; De Villiers, y Alexander, 2014; Khan et al., 2020).

La Tabla 6 muestra que ni el sector ni el tamaño se relacionaron significativamente con la información sobre aspectos de EC, por lo que rechazamos la H4 para ambas variables. Esto se puede explicar señalando que las empresas estudiadas no han desarrollado la EC como una estrategia y modelo de producción formal ni como práctica de desarrollo sostenible (Kirchherr et al., 2017; Veleva et al., 2017). El estudio encontró que son muy pocas las organizaciones que señalan la implementación de políticas de EC, solo algunas cumplen sus prácticas, pero no de forma sistemática ni como un proceso de innovación (Prieto-Sandoval et al., 2018). Esto también puede obedecer a la falta desarrollo de capacidades medioambientales de las empresas como prácticas de desempeño de sostenibilidad, contabilidad y reporte ambiental liderada por la dirección, así como una falta de influencia institucional y regulación para el desarrollo de sistemas de EC (Chiappetta, et al., 2020; Moktadir et al., 2020; Singhal, 2020). 


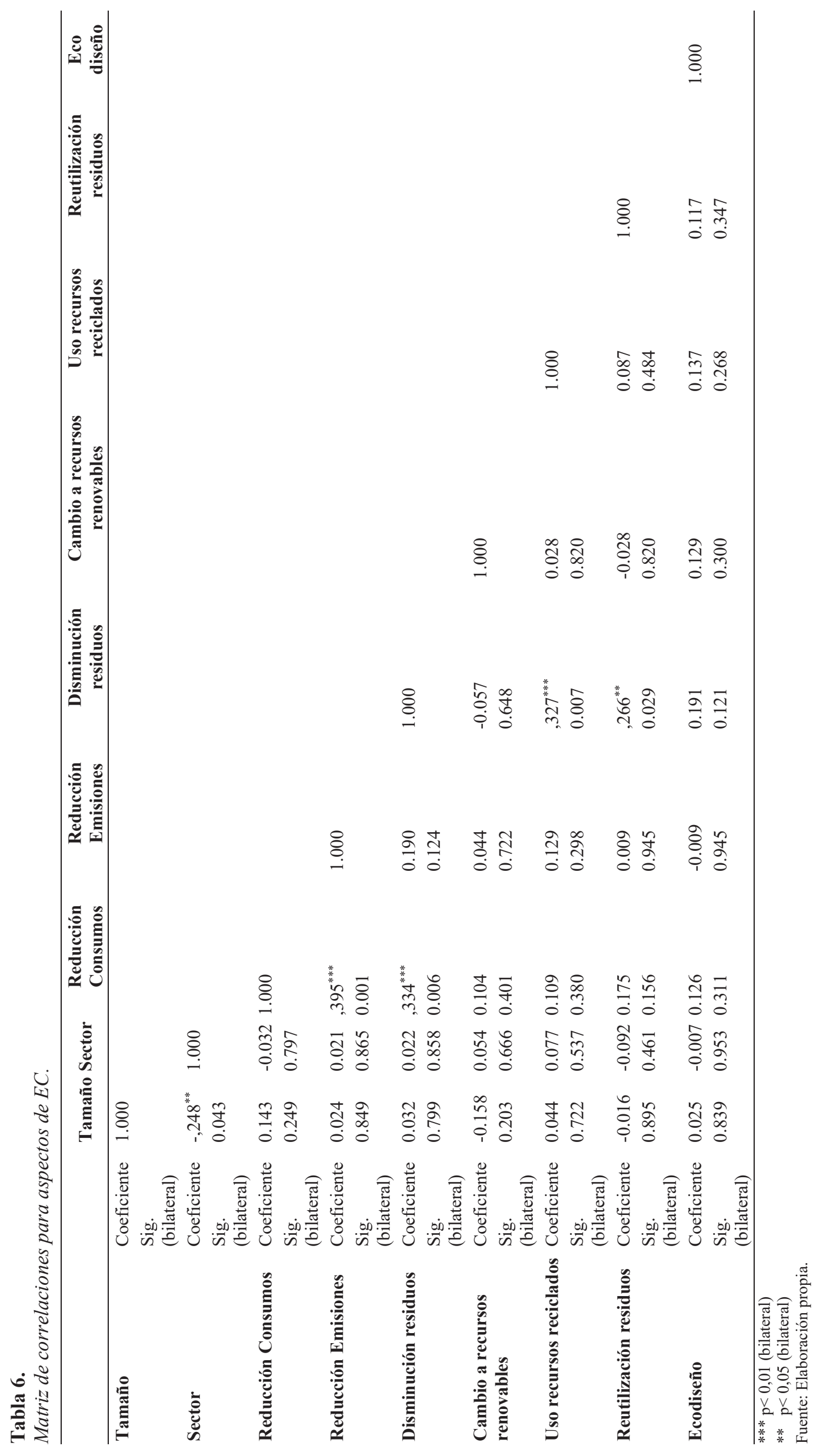




\section{Conclusiones}

Este estudio busca contribuir a la investigación de la naturaleza y factores de información medioambiental y de prácticas de $\mathrm{EC}$, divulgados en reportes de sostenibilidad de empresas peruanas. El reporte de información ambiental para la muestra estudiada no presenta un alto nivel de divulgación. Aquellas, de alto impacto de sostenibilidad, que presentan dicha información muestran una influencia institucional de estándares internacionales del GRI y de empresas similares de sus sectores como las mineras y de energía.

Por otro lado, grandes empresas que realizan actividades de agroindustria, industria, constructoras, de energía y mineras que muestran un uso intensivo de recursos y tienen un impacto en las comunidades donde operan, presentan un reporte de sostenibilidad que, en algunos casos, representa una forma de legitimación de sus actividades ante la influencia de los grupos de interés. En ese sentido, se informan aspectos de cumplimiento regulatorio, atención de reclamaciones e impacto en la biodiversidad. Asimismo, se deduce una influencia institucional de seguir similares prácticas de información basadas en estándares internacionales entre empresas del mismo sector, a diferencia de una menor influencia de promoción y regulaciones públicas.

Cabe destacar que se observa que la información de prácticas ambientales también se encuentra revelada por algunas empresas con menor tamaño como las empresas de prestación de servicios públicos y logística. Esto debe ser considerado un aporte de la investigación para que entidades no gubernamentales, asociaciones empresariales y el gobierno promuevan en todo tipo de organizaciones prácticas de desempeño y reporte ambiental, como parte de los objetivos de contribución al desarrollo sostenible y transparencia con los grupos de interés.

Respecto a las prácticas de $\mathrm{EC}$, los resultados señalan una falta de implantación de sistemas de EC en la gestión de sus actividades operativas. Aunque se encuentran algunas actividades de EC reveladas de forma separada, no son propiamente en la mayoría de casos partes de un sistema de gestión integral informado como tal en las memorias ni se incluyen como estrategias de desempeño e información ambiental lideradas por los directivos.
Resulta necesaria una mayor influencia institucional, mediante incentivos y regulaciones adecuadas por parte de entidades gubernamentales y privadas, para fomentar el desempeño ambiental y la implantación de modelos de negocio innovadores. La promoción de la EC representa una contribución relevante al desarrollo sostenible, y no solo una influencia institucional ni una forma de legitimación de actividades.

Este estudio ha presentado limitaciones de acceso a mayores reportes periódicos de sostenibilidad de empresas peruanas, por lo que se proponen estudios futuros de mayores reportes y en mayor periodo de tiempo, así como estudios cualitativos para evaluar la gestión de sostenibilidad en el contexto de las empresas.

\section{Referencias bibliográficas}

Aerts, W., Cormier, D., Magnan, M. (2006). Intraindustry imitation in corporate environmental reporting: An international perspective. Journal of Accounting and Public Policy, 25, 299-331. https://doi.org/10.1016/j. jaccpubpol.2006.03.004

Ali, W., y Frynas, J. G. (2018). The role of normative CSR-promoting institutions in stimulating CSR disclosures in developing countries. Corporate Social Responsibility and Environmental Management, 25(4), 373390. https://doi.org/10.1002/csr.1466

Alonso-Almeida, M., Marimon, F., y Llach, J. (2015). Difusión de las memorias de sostenibilidad en Latinoamérica: análisis territorial y sectorial. Estudios Gerenciales, 31, 139-149. https://doi.org/10.1016/j. estger.2015.01.002

Alvarez-Risco, A., Rosen, M. A., y Del-AguilaArcentales, S. (2020). A New Regulation for Supporting a Circular Economy in the Plastic Industry: The Case of Peru. Journal of Landscape Ecology, 13(1), 1-3. https://doi. org/10.2478/jlecol-2020-0004

Aranda-Usón, A., Portillo-Tarragona, P., Scarpellini, S., y Llena-Macarulla, F. (2020). The progressive adoption of a circular economy by businesses for cleaner production: An approach from a regional study in Spain. Journal of Cleaner Production, 247, 119648. https://doi.org/10.1016/j.jclepro.2019.119648

Baalouch, F., Damak, S., y Hussainet, K. (2019). A study of the determinants of environmental disclosure quality: evidence from French 
listed companies. Journal of Management and Governance, 23(4), 939-971. https://doi. org/10.1007/s10997-019-09474-0

Bebbington, J., Kirk, E. A., y Larrinaga, C. (2012). The production of normativity: A comparison of reporting regimes in Spain and the UK. Accounting, Organizations and Society, 37(2), 78-94. https://doi.org/10.1016/j. aos.2012.01.001

Bednárová, M., Klimko, R., y Rievajová, E. (2019). From Environmental Reporting to Environmental Performance. Sustainability, 11(9), 1-12. https://doi.org/10.3390/ su11092549

Bocken, N., De Pauw, I., Bakker, C., y Van der Grinten, B. (2016). Product design and business model strategies for a circular economy. Journal of Industrial and Production Engineering, 33(5), 308-320. https://doi.org/ $10.1080 / 21681015.2016 .1172124$

Calixto, L. (2013). A divulgação de relatórios de sustentabilidade na América Latina: um estudo comparativo. Revista de Administração, 48(4), 828-842. https://doi.org/10.5700/ rausp1124

Cerdá, E., y Khalilova, A. (2016). Economía circular. Economía industrial, 401, 11-20.

Chiappetta, J. C., Seuring, S., de Sousa Jabbour, A. B. L., Jugend, D., Fiorini, P. D. C., Latan, H., y Izeppi, W. C. (2020). Stakeholders, innovative business models for the circular economy and sustainable performance of firms in an emerging economy facing institutional voids. Journal of environmental management, 264, 110416. https://doi. org/10.1016/j.jenvman.2020.110416

Cormier, D., Magnan, M., y Van Velthoven, B. (2005). Environmental disclosure quality in large German companies: economic incentives, public pressures or institutional conditions?. European accounting review, 14(1), 3-39. https://doi.org/10.1080/0963818 $\underline{042000339617}$

Cormier, D., y Magnan, M. (2003). Environmental reporting management: a continental European perspective. Journal of Accounting and public Policy, 22(1), 43-62. https://doi. org/10.1016/S0278-4254(02)00085-6

D'Amico, E., Coluccia, D., Fontana, S., y Solimene, S. (2016). Factors Influencing Corporate Environmental Disclosure. Business Strategy and the Environment. 25(3), 178-192. https:// doi.org/10.1002/bse.1865

Da Silva, S.M., y Aibar, B. (2011). Factores
Determinantes del grado de información medioambiental divulgada en las grandes empresas que operan en Portugal: Un análisis univariante. Revista de Administração e Contabilidade da Unisinos, 8(1), 3-19.

Deegan, C. (2002). The legitimising effect of social and enviromental disclousure - a theoretical foundation. Accounting, Auditing \& Accountability Journal, 15(3), 282-311. https://doi.org/10.1108/09513570210435852

Del Barco, J. P., Del Barco, M. A., Del Barco, M. S., y Federici, I. (2018). Divulgación de aspectos ambientales en los informes de sostenibilidad frente al nuevo escenario internacional. CAPIC REVIEW, 16, 1-19. https://doi.org/10.35928/cr.vol16.2018.67

De Villiers, C., y Alexander, D. (2014). The institutionalisation of corporate social responsibility reporting. The British Accounting Review, 46(2), 198-212. https:// doi.org/10.1016/j.bar.2014.03.001

Elia, V., Gnoni, M.G., y Tornese, F. (2017). Measuring circulareconomy strategies through index methods: A critical analysis. Journal of Cleaner Production, 142, 2741-2751 https:// doi.org/10.1016/j.jclepro.2016.10.196

Ernst \& Young. 2016. "Value of sustainability reporting: a study by EY and Boston College Center for Corporate Citizenship". https:// www.ey.com/Publication/vwLUAssets/EY Value of Sustainability_Reporting/\%24File/ EY-Sustainability.pdf

Gallego-Álvarez, I., Belén, M., y Rodríguez, M. (2018). An analysis of the environmental information in international companies according to the new GRI standards. Journal of Cleaner Production. 182, 57-66. https:// doi.org/10.1016/i.jclepro.2018.01.240

Geng, Y., Fu, J., Sarkis, J., y Xue, B. (2012). Towards a national circular economy indicator system in China: an evaluation and critical analysis. Journal of Cleaner Production, 23, 216-224. https://doi.org/10.1016/j.jclepro.2011.07.005

Ghisellini, P., Cialani, C., Ulgiati, S. (2016). A review on circular economy: the expected transition to a balanced interplay of environmental and economic systems. Journal of Cleaner Production, 114, 11-32. https://doi.org/10.1016/j.jclepro.2015.09.007

Hernández-Pajares J. (2018). Influencia de la naturaleza internacional de empresas peruanas en su información de sostenibilidad. Revista de Comunicación 17(1). 74-92 https://doi. org/10.26441/RC17.1-2018-A4 
Husillos, J. (2007). Una aproximación desde la teoría de la legitimidad a la información medioambiental revelada por las empresas españolas cotizadas. Spanish Journal of Finance and Accounting, 36(133), 97-121. https://doi.org/10.1080/02102412.2007.1077 $\underline{9616}$

Jackson, G., Bartosch, J.,Avetisyan, E., Kinderman, D., y Knudsen, J. (2019). Mandatory nonfinancial disclosure and its influence on CSR: An international comparison. Journal of Business Ethics, 1-20. https://doi.org/10.1007/ s10551-019-04200-0

Jenkins, H., y Yakovleva, N. (2006). Corporate social responsibility in the mining industry: Exploring trends in social and environmental disclosure. Journal of cleaner production, 14(3-4), 271-284. https://doi.org/10.1016/j. jclepro.2004.10.004

Khan, M., Lockhart, J., y Bathurst, R. (2020). A multi-level institutional perspective of corporate social responsibility reporting: A mixed-method study. Journal of Cleaner Production, 265, $121739 . \quad$ https://doi. org/10.1016/j.jclepro.2020.121739

Kirchherr, J., Reike, D., y Marko, H. (2017). Conceptualizing the circular economy: An analysis of 114 definitions. Resources, Conservation \& Recycling, 127, 221-232. https://doi.org/10.1016/j. resconrec.2017.09.005

Landrum, N. E., y Ohsowski, B. (2018). Identifying worldviews on corporate sustainability: a content analysis of corporate sustainability reports. Business Strategy and the Environment, 27(1), 128-151. https://doi. org/10.1002/bse.1989

Larrinaga, C., Carrasco F., Correa C., Llena F., y Moneva J.M. (2002). Accountability and Accounting Regulation: The Case of the Spanish Environmental Disclosure Standard. European Accounting Review ,11(4), 723-740. https://doi. org/10.1080/0963818022000001000

Laso, J., García-Herrero, I., Margallo, M., VázquezRowe, I., Fullana, P., Bala, A., Gazullad, C. Irabiena, A., Aldacoa R. (2018). Finding an economic and environmental balance in value chains based on circular economy thinking: An eco-efficiency methodology applied to the fish canning industry. Resources, Conservation and Recycling, 133, 428-437. https://doi. org/10.1016/j.resconrec.2018.02.004
Li, H., Bao, W., Xiu, C., Zhang, Y., y Xu, H. (2010). Energy conservation and circular economy in China's process industries. Energy, 35(11), 4273-4281. https://doi.org/10.1016/j. energy.2009.04.021

Llena, F., Moneva, J. M., y Hernandez, B. (2007). Environmental disclosures and compulsory accounting standards: the case of Spanish annual reports. Business strategy and the Environment, 16(1), 50-63. https://doi. org $/ 10.1002 /$ bse. 466

Madein,A. (2020). The institution of environmental reporting in Japan: an exploratory study. AsiaPacific Management Accounting Journal, 15(3), 189-218. http://dx.doi.org/10.24191/ apmaj.v15i3.1316

Marimon, F. Alonso-Almeida, M., Rodríguez, M., y Alejandro, K. (2012). The worldwide diffusion of the global reporting initiative: what is the point?. Journal of Cleaner Production, 33, 132-144. https://doi. org/10.1016/j.jclepro.2012.04.017

Martínez-Ferrero, J., y García-Sánchez, I. M. (2017). Coercive, normative and mimetic isomorphism as determinants of the voluntary assurance of sustainability reports. International Business Review, 26(1), 102-118. https://doi.org/10.1016/j. ibusrev.2016.05.009

Mio, C., Fasan, M., Marcon, C., y Panfilo, S. (2020). The predictive ability of legitimacy and agency theory after the implementation of the EU directive on non-financial information. Corporate Social Responsibility and Environmental Management, 27(6), 2465-2476. https://doi.org/10.1002/csr.1968

Moneva, J.M., Portillo-Tarragona, P., LlenaMacarulla, F., y Scarpellini, S. (2018). Perspectivas e impacto de la Economía Circular en Aragón desde la óptica empresarial. Economía aragonesa, 66, 111-126.

Moneva, J.M., Jara-Sarrúa, L., Hernández-Pajares, J., y Del Barco, J. (2019). The disclosure of social issues in Latin American sustainability reports: an exploration of their disclosure in Argentina, Chile, and Peru. En Corporate Social Responsibility Disclosure and Assurance: A Growing Market. Cambridge Scholars Publishing, 135-174.

Moktadir, M. A., Kumar, A., Ali, S. M., Paul, S. K., Sultana, R., y Rezaei, J. (2020). Critical success factors for a circular economy: Implications for business strategy and the 
environment. Business strategy and the environment, 29(8), 3611-3635. https://doi. org/10.1002/bse. 2600

Neu, D., Warsame, H. and Pedwell, K. (1998). Managing public impressions: environmental disclosures in annual reports. Accounting, Organizations and Society, 23(3), 265$282 . \quad$ https://doi.org/10.1016/S03613682(97)00008-1

Orazalin, N.,y Mahmood., M.(2019). Determinants of GRI-based sustainability reporting: evidence from an emerging economy. Journal of Accounting in Emerging Economies. 10 (1),140-164. https://doi.org/10.1108/JAEE12-2018-0137

Potting, J., Hekkert, M., Worrell, E., y Hanemaaijer, A. (2017). Circular economy: measuring innovation in the product chain. PBL Netherlands Environmental Assessment Agency. $\quad$ https://dspace.library.uu.nl/ handle/1874/358310

Prieto-Sandoval, V., Jaca, C., y Ormazabal, M. (2018). Towards a consensus on the circular economy. Journal of Cleaner Production, 179, 605-615. https://doi.org/10.1016/j. jclepro.2017.12.224

Rabasedas, M. (2018). Divulgación de información medioambiental: análisis exploratorio para las entidades cotizadas en Argentina. CAPIC REVIEW, 16, 1-16. https://doi.org/10.35928/ cr.vol16.2018.63

Scarpellini, S., Marín-Vinuesa,L.M.,Aranda-Usón, A., y Portillo-Tarragona, P. (2020a). Dynamic capabilities and environmental accounting for the circular economy in businesses. Sustainability Accounting, Management and Policy Journal. 11(7), 1129-1158. https://doi. org/10.1108/SAMPJ-04-2019-0150

Scarpellini, S., Valero-Gil, J., Moneva, J. M., y Andreaus, M. (2020b). Environmental management capabilities for a "circular eco-innovation". Business Strategy and the Environment, 29(5), 1850-1864. https://doi. org/10.1002/bse. 2472
Simoni, L., Bini, L., y Bellucci, M. (2020). Effects of social, environmental, and institutional factors on sustainability report assurance: Evidence from European countries. Meditari Accountancy Research, 28(6), 1059-1087. https://doi.org/10.1108/ MEDAR-03-2019-0462

Singhal, D., Tripathy, S., y Jena, S. K. (2020). Remanufacturing for the circular economy: Study and evaluation of critical factors. Resources, Conservation and Recycling, 156, 104681. $\quad$ https://doi.org/10.1016/j. resconrec.2020.104681

Stewart, R., y Niero, M. (2018). Circular economy in corporate sustainability strategies: A review of corporate sustainability reports in the fastmoving consumer goods sector. Business Strategy and the Environment, 27(7), 10051022. https://doi.org/10.1002/bse.2048

Veleva, V., Bodkin, G., y Todorova, S. (2017). The need for better measurement and employee engagement to advance a circular economy: Lessons from Biogen's “zero waste" journey. Journal of cleaner production, 154, 517-529. https://doi.org/10.1016/j.jclepro.2017.03.177

Wirth, H., Kulczycka, J., Hausner, J., y Koński, M. (2016). Corporate Social Responsibility: Communication about social and environmental disclosure by large and small copper mining companies. Resources Policy, 49, 53-60. https://doi.org/10.1016/j. resourpol.2016.04.007

Ying, J., y Li-jun, Z. (2012). Study on Green Supply Chain Management Based on Circular Economy. Physics Procedia, 25, 1682-1688. https://doi.org/10.1016/j.phpro.2012.03.295

Yuan, Z., Bi, J., y Moriguichi, Y. (2006). The circular economy: A new development strategy in China. Journal of Industrial Ecology, 10(1-2), 4-8. https://doi. org/10.1162/108819806775545321 